\title{
Science highlights from Hipparcos
}

\author{
M. A. C. Perryman \\ European Space Agency, ESTEC, Noordwijk, The Netherlands
}

\begin{abstract}
The Hipparcos and Tycho Catalogues were published in 1997. In the intervening 10 years, several thousand papers making more-or-less direct use of the data have been published. I summarise a number of scientific applications which illustrate the variety of problems to which the data have been applied. This includes the re-reduction of old and contemporary astrometric observations, investigations of Galactic structure and dynamics, investigations of stellar structure and evolution, investigations of the Solar System and our Earth's environment, and various uses of the binary star and photometric data.
\end{abstract}

Keywords. astrometry, Galaxy: general, stars: general

\section{Introduction}

The fundamental task of measuring stellar positions, and the derived properties of distances and space motions, has preoccupied astronomers for centuries. As one of the oldest branches of astronomy, astrometry is concerned with measurement of the positions and motions of planets and other bodies within the Solar System, of stars within our Galaxy and, at least in principle, of galaxies and clusters of galaxies within the Universe as a whole. Accurate star positions provide a celestial reference frame for representing moving objects, and for relating phenomena at different wavelengths. Determining the systematic displacement of star positions with time gives access to their motions through space. Determining their apparent annual motion as the Earth moves in its orbit around the Sun gives access to their distances through measurement of parallax. All of these quantities, and others, are accessed from high-accuracy measurements of the relative angular separation of stars. Repeated measurements over a long-period of time provide the a stereoscopic map of the stars and their kinematic motions.

What follows, either directly from the observations or indirectly from modeling, are absolute physical stellar characteristics: stellar luminosities, radii, masses, and ages; and their dynamical signatures. The physical parameters are then used to understand their internal composition and structure, to disentangle their space motions and, eventually, to explain in a rigorous and consistent manner how the Galaxy was originally formed, and how it will evolve in the future. Crucially, stellar motions through space reflect dynamical perturbations of all other matter, visible or invisible.

Astrometry is in principle capable of measuring a whole host of higher-order phenomena: at the milliarcsec level, binary star signatures, General Relativistic light bending, and the dynamical consequences of dark matter are already evident; at the microarcsec level, targeted by the next generation of space astrometry missions currently under development, direct distance measurements will be extended across the Galaxy and to the Large Magellanic Cloud. Other effects will become routinely measurable at the same time: perspective acceleration and secular parallax evolution, more subtle metric effects, planetary perturbations of the photocentric motion, and astrometric micro-lensing; and at the nanoarcsec level, currently no more than an experimental concept, effects of optical interstellar scintillation, geometric cosmology, and ripples in space-time due to 
gravitational waves will become apparent. The bulk of this seething motion is largely below current observational capabilities, but it is there, waiting to be investigated.

The Hipparcos satellite was a space mission primarily targeting the uniform acquisition of milliarcsec-level astrometry (positions, parallaxes and annual proper motions) for some 120000 stars. The satellite was launched in August 1989 and operated until 1993. The data processing was finalised in 1996, and the results published by ESA in June 1997 as a compilation of 17 hard-bound volumes, a celestial atlas, and 6 CDs, comprising the Hipparcos and Tycho Catalogues. The original Tycho Catalogue comprised some 1 million stars of lower accuracy than the Hipparcos Catalogue, while the Tycho 2 Catalogue, published in 2000, provided positions and accurate proper motions for some 2.5 million objects, through the combination of the satellite data with plate material from the early 1900s. Details of the satellite operation, and the successive steps in the data analysis, and in the validation and description of the detailed data products, are included in the published catalogue. The result have been in the scientific domain for 10 years.

\section{The Hipparcos science}

The Hipparcos results impact a very broad range of astronomical research, which I will classify into three major themes:

\subsection{Provision of an accurate reference frame}

This has allowed the consistent and rigorous re-reduction of a wide variety of historical and present-day astrometric measurements. The former category include Schmidt plate surveys, meridian circle observations, 150 years of Earth-orientation measurements, and re-analysis of the 100-year old Astrographic Catalogue (and the associated Carte du Ciel). The Astrographic Catalogue data, in particular, have yielded a dense reference framework reduced to the Hipparcos reference system propagated back to the early 1900s. Combined with the dense framework of 2.5 million star mapper measurements from the satellite, this has yielded the high-accuracy long-term proper motions of the Tycho 2 Catalogue.

The dense network of the Tycho 2 Catalogue has, in turn, provided the reference system for the reduction of current state-of-the-art ground-based survey data: thus the dense UCAC 2 and USNO B2 catalogues are now provided on the same reference system, and the same is true for recent surveys such as SDSS and 2MASS. Other observations specifically reduced to the Hipparcos system are the SuperCOSMOS Sky Survey, major historical photographic surveys such as the AGK2 and the $\mathrm{CPC} 2$, and the more recent proper motion programmes in the northern and southern hemispheres, NPM and SPM. Proper motion surveys have been rejuvenated by the availability of an accurate optical reference frame, and amongst them are the revised NLTT (Luyten Two-Tenths) survey, and the Lépine-Shara proper motion surveys (north and south). Many other proper motion compilations have been generated based on the Hipparcos reference system, in turn yielding large data sets valuable for open cluster surveys, common-proper motion surveys, etc..

The detection and characterisation of double and multiple stars has been revolutionised by Hipparcos: in addition to binaries detected by the satellite, many others have been revealed through the difference between the Hipparcos (short-term) proper motion, and the long-term photocentric motion of long-period binary stars (the $\Delta \mu$ binaries). New binary systems have been followed up through speckle and long-baseline optical interferometry from ground, through a re-analysis of the Hipparcos Intermediate Astrometric Data, or through a combined analysis of astrometric and ground-based radial velocity data. Other binaries have been discovered as common-proper motions systems in cat- 
alogues reaching fainter limiting magnitudes. I have compiled a list of some 25 papers together revising the analysis of more than 15000 Hipparcos binary systems, providing new orbital solutions, or characterising systems which were classified by Hipparcos as suspected double, acceleration solutions, or stochastic ('failed') solutions.

I have also compiled a list of more than 30 papers together presenting radial velocities for more than 17000 Hipparcos stars since the catalogue publication, not counting two papers presenting some 20000 radial velocities from the CORAVEL data base, and some 25000 RAVE measurements including some Tycho stars. These radial velocity measurements are of considerable importance for determining the three-dimensional space motions, as well as further detecting and characterising the properties of binary stars.

More astrophysically, studies have been made of wide binaries, and their use as tracers of the mass concentrations during their Galactic orbits, and according to population. Numerous papers deal with improved mass estimates from the spectroscopic eclipsing systems, important individual systems such as the Cepheid-binary Polaris, the enigmatic Arcturus, and favourable systems for detailed astrophysical investigations such as V1061 Cyg, HIP 50796, the mercury-manganese star $\phi$ Her, and the spectroscopic binary HR 6046. A number of papers have determined the statistical distributions of periods and eclipse depths for eclipsing binaries, and others have addressed their important application in determining the radiative flux and temperature scales. Studies of the distributions of detached and contact binaries (including W UMa and symbiotic systems) have also been undertaken.

The accurate reference frame has in turn provided results in topics as diverse as the measurement of General Relativistic light bending; Solar System science, including mass determinations of minor planets; applications of occultations and appulses; studies of Earth rotation and Chandler Wobble over the last 100 years based on a re-analysis of data acquired over that period within the framework of studies of the Earth orientation; and consideration of non-precessional motion of the equinox.

\subsection{Constraints on stellar evolutionary models}

The accurate distances and luminosities of 100000 stars has provided the most comprehensive and accurate data set relevant to stellar evolutionary modeling to date, providing new constraints on internal rotation, element diffusion, convective motions, and asteroseismology. Combined with theoretical models it yields evolutionary masses, radii, and stellar ages of large numbers and wide varieties of stars.

A substantial number of papers have used the distance information to determine absolute magnitude as a function of spectral type, with new calibrations extending across the HR diagram: for example for OB stars, AFGK dwarfs, and GKM giants, with due attention given to the now more-quantifiable effects of Malmquist and Lutz-Kelker biases. Other luminosity calibrations have used spectral lines, including the Ca II-based Wilson-Bappu effect, the equivalent width of $\mathrm{O} \mathrm{I}$, and calibrations based on interstellar lines.

A considerable Hipparcos-based literature deals with all aspects of the basic 'standard candles' and their revised luminosity calibration. Studies have investigated the Population I distance indicators, notably the Mira variables, and the Cepheid variables (including the period-luminosity relation, and their luminosity calibration using trigonometric parallaxes, Baade-Wesselink pulsational method, main-sequence fitting, and the possible effects of binarity). The Cepheids are also targets for Galactic kinematic studies, tracing out the Galactic rotation, and also the motion perpendicular to the Galactic plane.

Hipparcos has revolutionised the use of red clump giants as distance indicators, by providing accurate luminosities of hundreds of nearby systems, in sufficient detail that 
metallicity and evolutionary effects can be disentangled, and the objects then used as single-step distance indicators to the LMC and SMC, and the Galactic bulge. Availability of these data has catalysed the parallel theoretical modeling of the clump giants.

For the Population II distance indicators, a rather consistent picture has emerged in recent years based on subdwarf main sequence fitting, and on the various estimates of the horizontal branch and RR Lyrae luminosities.

A number of different methods now provide distance estimates to the Large Magellanic Cloud, using both Population I and Population II tracers, including some not directly dependent on the Hipparcos results (such as the geometry of the SN 1987A light echo, orbital parallaxes of eclipsing binaries, globular cluster dynamics, and white dwarf cooling sequences). Together, a rather convincing consensus emerges, with a straight mean of several methods yielding a distance modulus of $(m-M)_{0}=18.49$ mag. Through the Cepheids, the Hipparcos data also provide good support for the value of the Hubble Constant $H_{0}=72 \pm 8 \mathrm{~km} \mathrm{~s}^{-1} \mathrm{Mpc}^{-1}$ as derived by the HST key project, and similar values derived by WMAP, gravitational lensing experiments, and Sunyaev-Zel'dovich effect.

A huge range of other studies has made use of the Hipparcos data to provide constraints on stellar structure and evolution. Improvements have followed in terms of effective temperatures, metallicities, and surface gravities. Bolometric corrections for the $H p$ photometric band have become available within the last few months through the work of Michael Bessell, opening the way for new and improved studies of the observational versus theoretical Hertzsprung-Russell diagram.

Many stellar evolutionary models have, of course, been developed and refined over the last few years, and Hipparcos provides an extensive testing ground for their validation and their astrophysical interpretation: these include specific models for pre- and postmain sequence phases, and models which have progressively introduced effects such as convective overshooting, gravitational settling, rotation effects, binary tidal evolution, radiative acceleration, and effects of $\alpha$-element abundance variations. These models have been applied to the understanding of the HR diagram for nearby stars, the reality of the Böhm-Vitense gaps, the zero-age main sequence, the subdwarf main sequence, and the properties of later stages of evolution: the subgiant, first ascent and asymptotic giant branch, the horizontal branch, and the effects of dredge-up, and mass-loss. Studies of elemental abundance variations include the age-metallicity relation in the solar neighbourhood, and various questions related to particular elemental abundances such as lithium and helium. Other studies have characterised and interpreted effects of stellar rotation, surface magnetic fields, and observational consequences of asteroseismology, notably for solar-like objects, the high-amplitude $\delta$ Scuti radial pulsators, the $\beta$ Cephei variables, and the rapidly-oscillating Ap stars.

Many studies have focused on the pre-main sequence stars, both the (lower-mass) $\mathrm{T}$ Tauri and the (higher-mass) Herbig Ae/Be stars, correlating their observational dependencies on rotation, X-ray emission, etc. The understanding of Be stars, chemicallypeculiar stars, X-ray emitters, and Wolf-Rayet stars have all been substantially effected by the Hipparcos data. Kinematic studies of runaway stars, produced either by supernova explosions or dynamical cluster ejection, have revealed many interesting properties of runaway stars, also connected with the problem of (young) B stars found far from the Galactic plane.

Dynamical orbits within the Galaxy have been calculated for planetary nebulae and, perhaps surprisingly given their large distances, for globular clusters, where the provision of a reference frame at the 1 milliarcsec accuracy level has allowed determination of their space motions and, through the use of a suitable Galactic potential, their Galactic orbits, 
with some interesting implications for Galactic structure, cluster disruption, and Galaxy formation.

One of the most curious of the Hipparcos results in this area is probably the improved determination of the empirical mass-radius relation of white dwarfs. At least three such objects appear to be too dense to be explicable in terms of carbon or oxygen cores, while iron cores seem difficult to generate from evolutionary models. 'Strange matter' cores have been postulated, and studied by a number of theoretical groups.

\subsection{Galactic structure and dynamics}

The distances and uniform space motions have provided a substantial advance in understanding of the detailed kinematic and dynamical structure of the solar neighbourhood, ranging from the presence and evolution of clusters, associations and moving groups, the presence of resonance motions due to the Galaxy's central bar and spiral arms, the parameters describing Galactic rotation, the height of the Sun above the Galactic midplane, the discrimination of the motions of the thin disk, thick disk and halo populations, and the evidence for halo accretion.

Many attempts have been made to further understand and characterise the solar motion based on Hipparcos data, and to redefine the large-scale properties of Galactic rotation in the solar neighbourhood. The latter has been traditionally described in terms of the Oort constants, but it is now evident that such a formulation is quite unsatisfactory in terms of describing the detailed local stellar kinematics. Attempts have been made to re-cast the problem into the 9-component tensor treatment of the Ogorodnikov-Milne formulation, analogous to the treatment of a viscous and compressible fluid by Stokes more than 150 years ago. The results of several such investigations have proved perplexing. The most recent and innovative approach has been a kinematic analysis based on vectorial harmonics, in which the velocity field is described in terms of (some unexpected) 'electric' and 'magnetic' harmonics. They reveal the warp at the same time, but in an opposite sense to the vector field expected from the stationary warp model.

Kinematic analyses have tackled the issues of the mass density in the solar neighbourhood, and the associated force-law perpendicular to the plane, the $K_{z}$ relation. Estimates of the resulting vertical oscillation frequency in the Galaxy of around 80 Myr have been linked to cratering periodicities in the Earth's geological records. Related topics include studies of nearby stars, the stellar escape velocity, the associated initial mass function, and the star formation rate over the history of the Galaxy. Dynamical studies of the bar, of the spiral arms, and of the stellar warp, have all benefited. Studies of the baryon halo of our Galaxy have refined its mass and extent, its rotation, shape, and velocity dispersion, and have provided compelling evidence for its formation in terms of halo substructure, some of which is considered to be in falling, accreting material, still ongoing today.

New techniques have been developed and refined to search for phase-space structure (i.e. structure in positional and velocity space): these include convergent-point analysis, the spaghetti method of Hoogerwerf \& Aguilar, global convergence mapping, epicycle correction, and orbital back-tracking. An extensive literature has resulted on many aspects of the Hyades, the Pleiades, and other nearby open clusters, comprehensive searches for new clusters, and their application to problems as diverse as interstellar reddening determination, correlation with the nearby spiral arms, and the age dependence of their vertical distribution within the galaxy: one surprising result is that this can be used to place constraints on the degree of convective overshooting by matching stellar evolutionary ages with cluster distances from the Galactic plane.

In addition to studying and characterising open clusters, and young nearby associations of recent star formation, the Hipparcos data have revealed a wealth of structure in the 
nearby velocity distribution which is being variously interpreted in terms of open cluster evaporation, resonant motions due to the central Galactic bar, scattering from nearby spiral arms, and the effects of young nearby kinematic groups, with several having been discovered from the Hipparcos data in the last 5 years.

The Hipparcos stars have been used as important (distance) tracers, determining the extent of the local 'bubble', itself perhaps the result of one or more nearby supernova explosions in the last $5 \mathrm{Myr}$. The interstellar medium morphology, extinction and reddening, grey extinction, polarisation of star light, and the interstellar radiation field, have all been constrained by the Hipparcos stars.

\subsection{Other applications}

Superficially it may seem surprising that the Hipparcos Catalogue has been used for a number of studies related to the Earth's climate. Studies of the passage of nearby stars and their possible interaction with the Oort Cloud have identified stars which came close to the Sun in the geologically recent past, and others which will do so in the relatively near future. Analysis of the Sun's Galactic orbit, and its resulting passage through the spiral arms, favour a particular spiral arm pattern speed in order to place the Sun within these arms during extended deep glaciation epochs in the distant past. In this model, climatic variations are explained as resulting from an enhanced cosmic-ray flux in the Earth's atmosphere, leading to cloud condensation and a consequent lowering of temperature. A study of the Maunder Minimum, a period between 1645-1715 coinciding with the coldest excursion of the 'Little Ice Age', and a period of great hardship in Europe, was interpreted in the context of the number of solar-type stars out to 50$80 \mathrm{pc}$ showing correspondingly decreased surface activity. Several studies have used the accurate distance data, accompanied by stellar evolutionary models, in an attempt to identify 'solar twins' (stars which most closely resemble the Sun in all its characteristics, and which may be the optimum targets for searches for life in the future), and 'solar analogues' (stars which will resemble the Sun at some past or future epoch, and which therefore offer the best prospects for studying the Sun at different evolutionary stages).

Many studies have used the accurate photometric data: as part of the construction of absolute or bolometric magnitudes, for their uniform colour indices, and for their extensive epoch photometry, which itself has been used for all sorts of variability analyses, including the rotation of minor planets, the study of eclipsing binaries, the complex pulsational properties of Cepheids, Mira variables, $\delta$ Scuti, slowly-pulsating B stars, and many others.

In addition to all of these, the Hipparcos and Tycho Catalogues are now routinely used to point ground-based telescopes, navigate space missions, drive public planetaria, and provide search lists for programmes such as exo-planet surveys; one study has even shown how positions of nearby stars at the milliarcsec level can be used to optimise search strategies for extraterrestrial intelligence.

\section{The full review}

This short synopsis provides an interim report of a full review, which will be published by Cambridge University Press in mid-2008. Titled 'Astronomical Applications of Astrometry: A Review Based on Ten Years of Exploitation of the Hipparcos Data', this book-length review covers the full range of the Hipparcos scientific findings, in an extensive summary and analysis of the scientific literature over the 10 years since the publication of the Hipparcos and Tycho Catalogues in 1997. 